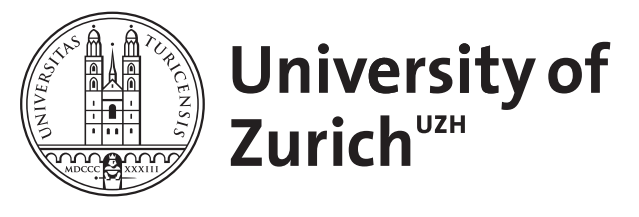

\title{
Polarizing optics in a spider eye
}

\author{
Mueller, K P ; Labhart, T
}

\begin{abstract}
Many arthropods including insects and spiders exploit skylight polarization for navigation. One of the four eye pairs of the spider Drassodes cupreus is dedicated to detect skylight polarization. These eyes are equipped with a tapetum that strongly plane-polarizes reflected light. This effectively enhances the polarization-sensitivity of the photoreceptors, improving orientation performance. With a multidisciplinary approach, we demonstrate that D. cupreus exploits reflective elements also present in non-polarizing tapetal eyes of other species such as Agelena labyrinthica. By approximately orthogonal arrangement of two multilayer reflectors consisting of reflecting guanine platelets, the tapetum uses the mechanism of polarization by reflection for polarizing reflected light.
\end{abstract}

DOI: https://doi.org/10.1007/s00359-010-0516-6

Posted at the Zurich Open Repository and Archive, University of Zurich

ZORA URL: https://doi.org/10.5167/uzh-33570

Journal Article

Published Version

Originally published at:

Mueller, K P; Labhart, T (2010). Polarizing optics in a spider eye. Journal of Comparative Physiology A, 196(5):335-348.

DOI: https://doi.org/10.1007/s00359-010-0516-6 


\title{
Polarizing optics in a spider eye
}

\author{
Kaspar P. Mueller • Thomas Labhart
}

Received: 6 January 2010/Revised: 24 February 2010/Accepted: 25 February 2010/Published online: 14 March 2010

(C) Springer-Verlag 2010

\begin{abstract}
Many arthropods including insects and spiders exploit skylight polarization for navigation. One of the four eye pairs of the spider Drassodes cupreus is dedicated to detect skylight polarization. These eyes are equipped with a tapetum that strongly plane-polarizes reflected light. This effectively enhances the polarization-sensitivity of the photoreceptors, improving orientation performance. With a multidisciplinary approach, we demonstrate that $D$. cupreus exploits reflective elements also present in nonpolarizing tapetal eyes of other species such as Agelena labyrinthica. By approximately orthogonal arrangement of two multilayer reflectors consisting of reflecting guanine platelets, the tapetum uses the mechanism of polarization by reflection for polarizing reflected light.
\end{abstract}

Keywords Polarization vision - Drassodes cupreus .

Optics · Tapetum $\cdot$ Spiders

$\begin{array}{ll}\text { List of symbols } \\ R_{\mathrm{S}} & \begin{array}{l}\text { Reflection of light oscillating orthogonal to the } \\ \text { plane of incidence }\end{array} \\ R_{\mathrm{p}} & \begin{array}{l}\text { Reflection of light oscillating parallel to the } \\ \text { plane of incidence }\end{array} \\ P_{\mathrm{r}} & \begin{array}{l}\text { Degree of polarization } \\ \theta_{\mathrm{i}}\end{array} \\ & \begin{array}{l}\text { Angle of incidence on the reflector (relative to } \\ \text { the normal on the reflector) }\end{array}\end{array}$

K. P. Mueller $(\varangle) \cdot$ T. Labhart

Neuroscience Center Zurich and Center for Integrative Human Physiology, Institute of Molecular Life Sciences,

University of Zurich, Winterthurerstr. 190,

8057 Zurich, Switzerland

e-mail: kaspar.mueller@imls.uzh.ch

T. Labhart

e-mail: thomas.labhart@imls.uzh.ch $\alpha \quad$ Angle of illumination of the eye (relative to the symmetry plane in the long axis of the eye)

$\varphi \quad$ Angular orientation of e-vector

$n_{1} \quad$ Refractive index of low index material

$n_{\mathrm{h}} \quad$ Refractive index of isotropic high index material

$n_{\mathrm{x}}, n_{\mathrm{y}}, n_{\mathrm{z}} \quad$ Refractive indices of birefringent high index material

$n_{\mathrm{a}} \quad$ Refractive index of air

$n_{\mathrm{c}} \quad$ Refractive index of cornea

\section{Introduction}

Polarized skylight provides polarization-sensitive organisms with a useful reference for a visual compass, which can be employed for navigation tasks in the context of path integration, or for keeping a cruising course during a journey (Wehner and Labhart 2006). Sunlight scattered in the atmosphere results in partially plane-polarized skylight. Following the rule of first-order Rayleigh scattering, as a first approximation the celestial polarization pattern is defined by the following principle: The prevailing oscillation plane (e-vector orientation) is oriented orthogonal to an imaginary straight line connecting an observed point in the sky with the sun (Strutt 1871; Suhai and Horváth 2004).

A prerequisite for polarization vision is an array of photoreceptors that are sensitive to e-vector orientation. The microvillar photoreceptors of arthropods are inherently polarization-sensitive because the dipole axes of the visual pigment molecules are aligned more or less parallel to the long axis of the microvilli. As a result, plane-polarized light is maximally absorbed when the e-vector orientation 
is parallel to the long axis of the microvillus axis (Israelachvili and Wilson 1976; Goldsmith and Wehner 1977; Hardie 1984). However, the high polarization sensitivity of the microvillus can be exploited by the whole photoreceptor only if the microvilli of the cell are exactly or nearly parallel to each other (avoiding randomizing effects) and if the rhabdom is reasonably short (avoiding self-screening) (Wehner et al. 1975; Nilsson et al. 1987). Such photoreceptors form the basis of polarization vision.

The neural mechanisms of polarized skylight navigation have been studied mainly in insects, where polarization vision appears to be mediated by just a small part of the compound eye, the dorsal rim area (DRA) (Labhart and Meyer 1999, 2002; Horváth and Varjú 2004; Wehner and Labhart 2006). The DRA has special properties: (1) the visual field of the DRA is directed to the sky; (2) the microvilli forming the rhabdoms of the photoreceptors are aligned parallel to each other, and consequently the receptors are strongly polarization sensitive; (3) in each ommatidium, the photoreceptors come in two sets having their microvilli oriented about $90^{\circ}$ to each other, i.e., they are tuned to mutually orthogonal e-vector orientations. This arrangement serves polarization-opponency, which further enhances polarization sensitivity, and discounts information on light intensity. Polarization vision has also been demonstrated for a number of spiders. Using behavioral observations, direct experimental or circumstantial evidence suggests that spiders also exploit skylight polarization for navigation (Papi 1955; Görner 1962; Henton and Crawford 1966; Dacke et al. 1999, 2001; Ortega-Escobar and Muñoz-Cuevas 1999).

Unlike insects, spiders are equipped with a set of (usually) eight simple eyes of the camera type (Homann 1971). As demonstrated in a number of lycosid and agelenid species, these spiders also use specialized photoreceptors for polarized skylight detection (Melamed and TrujilloCenóz 1966; Schröer 1974; Kovoor et al. 1993; Dacke et al. 2001). They are located in the ventral retina of the principal eyes and, thus, are directed skywards (Dacke et al. 2001). As in the insect dorsal rim area, all microvilli of a receptor cell have the same orientation, indicating strong polarization sensitivity. In addition, the ventral retina of these spiders contains two populations of cells with mutually orthogonal orientations of the microvilli.

In contrast to lycosids and agelenids, the gnaphosid spider Drassodes cupreus dedicates a complete pair of eyes to polarized skylight detection (Dacke et al. 1999). Situated on the dorsal surface of the prosoma, the postero-median (PM) eyes of this spider have common dorsal fields of view (Dacke et al. 2001). In each eye, the photoreceptor microvilli are oriented parallel to each other, but the microvillar orientation of the left and right eyes are perpendicular to each other. Thus, although morphologically different, the ventral part of the lycosid's principal eyes, and the secondary eyes of $D$. cupreus seem to be functionally analogous to the dorsal rim area of the insect compound eyes.

Unlike the principal eyes, the three pairs of secondary eyes in spiders are equipped with a tapetum (Homann 1971). The tapetum of the PM eyes of D. cupreus is of the canoe-shaped type consisting of two reflectors enclosing the photoreceptor cells. Light reflected by the tapetum is strongly plane-polarized, i.e., the tapetum acts as a polarizer (Dacke et al. 1999). Since the prevailing e-vector of the reflected light is oriented parallel to the microvilli of the receptors cells, the intrinsic polarization sensitivity is boosted, which in turn improves e-vector detection.

How does the polarizer work? In the present study, we hypothesize that the polarizing effect is based on a simple optical principle, the phenomenon of polarization by reflection. To test this hypothesis, we compared measurements of light reflection, carried out on intact eyes, with theoretical data calculated for a model tapetum. Thus, using micro-reflectometry we assessed the degree of polarization of reflected light for different angles of incidence and wavelengths. Providing the parameters for setting up the model, histological examinations revealed that the tapetum consists of two multilayer interference reflectors forming a V-shaped groove with an angle of approximately $95^{\circ}$ between them. Histochemical examinations and high-performance liquid chromatography/mass spectrometry (HPLC/MS) analyses indicated that the high refractive index layers of the multilayer stacks consist of guanine crystals. The comparison between optical and modeled data supports the thesis that the polarization of light returned by the tapetum results from a double reflection of incident light at the two reflectors of the $\mathrm{V}$-shaped tapetum, and no additional optical mechanisms need to be invoked.

\section{Materials and methods}

Animals

Adult D. cupreus (Blackwall, 1834) specimens (Araneae, Gnaphosidae) were collected near Baden (Switzerland). Spiders were found sitting in their silky nests under flat stones, where these nocturnal hunters spend the day. The spiders were kept in the laboratory at $22^{\circ} \mathrm{C}$ under long-day $(\mathrm{L}: \mathrm{D}=14: 10)$ conditions. They were placed individually in transparent polypropylene containers $(140 \times 105 \times$ 110 mm, EJS Verpackungen AG, Schüpfen, Switzerland) with a cast-in plaster ground, which was kept moist to maintain almost $100 \%$ air humidity. Small holes in the sides of the containers allowed air convection. The spiders 
built their nests under a piece of egg carton. They were fed Drosophila flies and cricket larvae twice a week. For comparative studies, Agelena labyrinthica (Clerck, 1757) spiders (Araneae, Agelenidae) were collected on hedges on the university campus and kept under the same conditions as D. cupreus spiders.

\section{Histology}

The spiders were anesthetized with $\mathrm{CO}_{2}$. The frontal part of the prosoma with the eyes was cut away with a razor blade and placed in cold $\left(4^{\circ} \mathrm{C}\right) 2.5 \%$ glutaraldehyde in $0.05 \mathrm{M}$ cacodylate-buffer (350 mOsm, pH 7.4). After 2 h fixation, the preparations were washed in cacodylate buffer at $4{ }^{\circ} \mathrm{C}$. Postfixation was at room temperature in $1.3 \% \mathrm{OsO}_{4}$ in S-collidine buffer (pH 7.4) for another $2 \mathrm{~h}$. After rinsing in distilled water, followed by dehydration in 2,2-dimethoxypropane and acetone, the eyes were embedded in EPON 812. The eyes were sectioned with a Reichert Ultramicrotome Om U3. Semi-thin $(2 \mu \mathrm{m})$ sections for light microscopy were stained with methylene blue. Ultrathin sections for electron microscopy were mounted on copper grids and either left unstained, stained with $0.7 \%$ uranyl acetate only or stained with $0.7 \%$ uranyl acetate and $2.5 \%$ lead citrate at room temperature. Sections were examined and photographed with a transmission electron microscope (Zeiss EM 109).

\section{Histochemistry}

Previous authors reported that the crystal platelets forming the reflective layer of the tapetum were broken away by mechanical pressure during histological sectioning (Setoguti 1967; Frese 1978). To make sure that the crystals were still in place, unstained sections were examined between crossed polarizers before histochemical examination. To identify the chemical nature of the crystals, which were earlier claimed to consist of guanine (Land 2000; Schwab et al. 2002), sections mounted on glass slides were treated with Schmorl's cyanoferrat method (Waldmann 1962) previously used to identify guanine: sections were stained in a $1: 1$ solution of $1 \% \mathrm{FeCl}_{3}$ and $1 \%$ potassium hexacyanoferrate(III) for $10 \mathrm{~min}$ and rinsed in distilled water.

\section{HPLC/MS-analysis}

Extracts of eyes were analyzed by means of high-pressure liquid chromatography with UV-diode array detector, online coupled to an electrospray ionization-mass spectrometry detector (HPLC-UV(DAD)-ESI-MS). Adult D. cupreus specimens were anesthetized by $\mathrm{CO}_{2}$ and the eyes were dissected out and extracted in one of three ways: (1) immersion in $100 \mu 10 \%$ trifluor acetic acid (TFA) under sonification at $50^{\circ} \mathrm{C}$ for $18 \mathrm{~h}$; (2) immersion in $50 \mu \mathrm{l} 10 \%$ TFA under sonification at $50^{\circ} \mathrm{C}$ for $8 \mathrm{~h}$; and (3) immersion in $50 \mu \mathrm{l} 10 \% \mathrm{TFA}$ at $4^{\circ} \mathrm{C}$ for $18 \mathrm{~h}$ (without sonification). Each sample either contained eyes with tapetum (anterolateral eyes: 1 sample; PM eyes: 1 sample; postero-lateral eyes: 1 sample; all secondary eyes: 4 samples) or tissue without tapetum (antero-median eyes: 4 samples; brain tissue: 1 sample). After centrifugation of the samples, $5 \mu \mathrm{l}$ liquid was injected into the HPLC/MS-system. Guanine and adenine solutions ( $1 \mathrm{ng} / \mu \mathrm{l}$ each) in 10\% TFA were injected as references. HPLC-ESI-MS analyses were performed on an Agilent 1100 HPLC system (Agilent Technologies, Palo Alto, CA, USA) fitted with a HTS PAL autosampler (CTC Analytics, Zwingen, Switzerland), an Agilent 1100 binary pump, and an Agilent 1100 photodiode-array detector. The details of chromatographic and MS conditions can be provided on request.

\section{Optical examinations}

Optical measurements were made with a Zeiss Photomicroscope II, provided with axial epi-illumination (Fig. 1a). A mercury short arc lamp (HBO; Osram HBO 50 W/AC) equipped with a heat absorbing filter (HF) served as light source. The wavelength range of the light was controlled by introducing broadband filters (SF) with $50 \mathrm{~nm}$ half-width (K40, K45, K50, K55 and K60, Balzers AG, Balzers, Liechtenstein) into the light path. Diaphragms (FD1) of different sizes in the light path were focused on the cornea in order to illuminate one whole eye (Fig. 1d) or part of an eye only (Fig. 1e). The intensity of light reflected from the eye was measured with a photometer (PD; Photodiode 222 AUV with Model 161 optometer, United Detector Technology, Hawthorne, USA). A diaphragm (FD2) in front of the photodiode separated light reflected by the eye from stray light (glare produced by the microscope's optics). A linear polarizer (analyzer; PA) in the exit beam could be rotated in order to measure the reflection intensity as a function of the plane of polarization. The microscope objective (OL) was a Zeiss Luminar $16 \mathrm{~mm} / 0.2$. To take photographs of the eye, the photodiode (PD), and the field diaphragm (FD2), were replaced by a digital camera (NIKON Coolpix 990, NIKON Corporation, Tokyo, Japan).

Live specimens of $D$. cupreus were fixed on a block of wax, mounted in a goniometer placed under the microscope, and the reflection intensities from the PM eyes were measured. The spiders were oriented such that the long axis of the observed eye (defined by the slit at the base of the canoe-shaped tapetum, refer to Fig. 1b) was oriented orthogonal to the plane formed by the light beam entering the microscope and the optical axis of the microscope (refer to Fig. 1a: the long axis of the schematic PM eye is oriented orthogonal to the drawing plane). To minimize the 


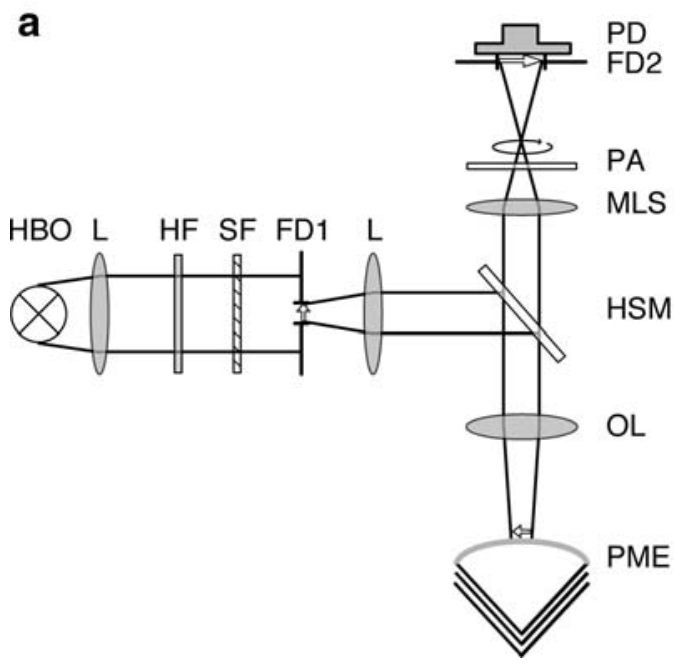

Fig. 1 Measuring light reflection from the tapetum of the posteromedian eyes in Drassodes cupreus. a Schematic drawing of light paths in the microscope used to measure reflected light. FD1, FD2 field diaphragms, $H B O$ mercury lamp, $H F$ heat-absorbing filter, $H S M$ half-silvered mirror, $L$ lenses, $M L S$ microscope lens system, $O L$ objective lens, $P A$ polarization analyzer, $P M E$ postero-median eye, $P D$ photodiode, $S F$ spectral filter. b Dorsal view of postero-median eyes illuminated with zenithal unpolarized light. Double arrow indicates e-vector orientation transmitted by the polarization analyzer (PA in a), white line indicates section planes in c and in Fig. 2; $R$ corneal reflection. Calibration bar $50 \mu \mathrm{m}$. c Schematic

influence of the (unpolarized) bright reflection of the incident light from the lens surface, the spider was slightly tilted forward or backward along the long-axis of the eye, such that the reflection from the lens moved out of the field of view as much as possible. Two types of measurements were performed: (1) measurements of reflection intensities as a function of the angle of incidence and (2) as a function of the wavelength of light.

\section{Angle-dependent measurements}

Reflection intensities were measured under different angles of illumination $\left(-45^{\circ}\right.$ to $+45^{\circ}$ with respect to the symmetry plane in the long axis of the eye; Fig. 1c) for green light (broadband spectral filter, $\lambda_{\max }=550 \mathrm{~nm}$ ). Maximal and minimal reflections were sought by adjusting the plane of polarization (by rotating the polarization analyzer), and the measured reflection intensities $\left(R_{\mathrm{md}}\right)$ together with the corresponding e-vector orientations $\varphi$ were noted. Measurements of light reflection from an aluminum mirror oriented orthogonal to the light beam revealed some polarization caused by the microscope's optics. Therefore, reflection intensities from the aluminum mirror $\left(R_{\mathrm{al}}\right)$ were measured at different e-vector orientations $\varphi$. Based on these data, a calibration curve was calculated that served to correct the measured reflection intensities of the eye: cross-section through the tapetum as indicated in b. Arrowed lines define angles of illumination relative to the symmetry plane of the tapetum. Note that the direction of a ray reflected by the tapetum depends on whether an incoming ray first hits the left or the right reflector (exemplified by the two different blue lines for the case of $\alpha=+15^{\circ}$ ). d, e Illuminating and measuring conditions (controlled by FD1 in a). d Illumination of whole eye width (included by yellow circle), resulting in reflection from both sides of the tapetum (light blue area). e Illumination of a small area on one side of the tapetum (yellow area), resulting in reflection from the other side (light blue area). The rest of the eye remains dark (dark blue areas)

$R_{\mathrm{cor}}=\frac{R_{\mathrm{md}}(\varphi)}{R_{\mathrm{al}}(\varphi)}$

From the corrected minimal and maximal intensities, the degree of polarization of reflected light $P_{\mathrm{r}}$ was calculated as:

$P_{r}=\frac{R_{\max }-R_{\min }}{R_{\max }+R_{\min }}$

It cannot be excluded that the intrinsic polarization by the microscope optics includes both linear and circular components. However, our polarimeter system and the spider's photoreceptors are both linear polarization analyzers and any circular components of the light beam are disregarded by both systems.

\section{Wavelength-dependent measurements}

Reflection intensities were measured under zenithal $\left(0^{\circ}\right)$ illumination using five broadband spectral filters with peak transmissions at 400, 450, 500, 550 and $600 \mathrm{~nm}$. Transmission spectra of the filters were recorded and convolved with the emission spectrum of the HBO lamp. From the resulting curves, the barycenter of the area under the curve was determined for each filter. The corresponding median wavelengths $\lambda_{\text {med }}$ were $370,440,500$, 550 and $580 \mathrm{~nm}$. The measured reflection intensities were 
corrected and the degree of polarization $P_{\mathrm{r}}$ was calculated as above.

\section{Data analysis}

Optical data were analyzed by repeated measures ANOVA tests with the two eyes of each individual and the angle of incidence or the wavelength of light, respectively, as within-subjects effects. Statistical analysis was conducted with SPSS 11.0.4 for Mac OS X.

\section{Mathematical modeling}

The equations used for calculating reflection by a birefringent multilayer stack as a function of the plane of polarization are given in "Appendix". From the reflection intensities of p- (light oscillating parallel to the plane of incidence) and s-polarized light (light oscillating orthogonal to the plane of incidence; refer to Fig. 6b), the degree of polarization was calculated as described above. Mathematical modeling of the reflective system was performed with MATLAB ${ }^{\circledR} 7.1 .0 .183$ R 14 Service Pack 3 with the aid of the "Electromagnetic waves and antenna" toolbox (Orfanidis 2008). The MATLAB code can be obtained on request.

\section{Results}

\section{Structure}

The PM eyes of D. cupreus lie close together on the dorsal surface of the prosoma pointing upwards. They have an oval shape and an angle of approximately $90^{\circ}$ between their long axes (Fig. 1b). In contrast, the PM eyes of A. labyrinthica have a circular shape (not shown), the long axes of their tapetal 'canoes' are approximately parallel to each other and the eyes point more sideways than the PM eyes of D. cupreus.

Confirming previous findings (Dacke et al. 1999), in tangential sections through the PM eyes of D. cupreus, a regular arrangement of the photoreceptor rhabdoms is observed. Almost all photoreceptors have their microvilli oriented parallel to the long axis of the eye; they were called "main receptors" (Dacke et al. 1999). In the middle of the eye along the long axis, there are two receptors with the microvilli orientated also parallel to the cornea but orthogonal to those of the main receptors. We call them "central receptors"; the previously used term "shallow receptors" (Dacke et al. 1999) is not justified because we found that these cells were not restricted to the distal retina (Fig. 2a: CR). The retina does not contain any screening pigment. In the PM eyes of $A$. labyrinthica, the arrangement of the receptors and their rhabdoms is much less regular (data not shown).

The lenses of the PM eyes of D. cupreus are not lenticular in shape but merely form slightly curved windows (Fig. 2a: L; Dacke et al. 1999). Since the lenses lack dioptric power, the eyes are incapable of producing a focused image on the retina and the tapetum can directly be observed in the intact eye without neutralizing the optics. In contrast, the PM eyes of $A$. labyrinthica have well developed lenses (Fig. 2b: L). The tapeta of the PM eyes in $D$. cupreus and A. labyrinthica belong to the canoe-shaped type (Homann 1971). In D. cupreus, the tapetum of the PM eyes consists of two approximately flat mirrors forming a V-shaped groove with an angle of approximately $95^{\circ}$ between the sides, as measured in light micrographs of cross sections through the eyes (mean $\pm \mathrm{SD} 94.6^{\circ} \pm$ $12.0^{\circ} ; 14$ spiders; Fig. 2a). In some eyes, one side of the tapetum was found to be slightly curved. It is not clear whether this is the native state or due to a slight deformation during the histological process. According to Dacke et al. (1999) the tapetum consists of "two flat mirrors... with an angle of $90^{\circ}$ to $100^{\circ}$ between the sides". In the PM eyes of $A$. labyrinthica, both sides of the tapetum were always curved forming a " $U$ " rather than a "V". In addition, the tapetum is less wide and thicker at some locations than in D. cupreus (Fig. 2b).

To assess the optical properties of the tapetum in the PM eyes of $D$. cupreus, electron micrographs of ultra-thin cross sections through the eye were analyzed. The tapetum is organized as a multilayer interference reflector in which alternating thin layers of high and low refractive index (1520 each) are stacked one upon another (Fig. 3a: HL, LL). Lying essentially parallel to each other, the arrangement of these layers is very regular (Fig. 2c). In a multilayer interference reflector, a portion of light is reflected at each interface of two layers (Fig. 3d).

The high refractive index layers of spider tapeta were proposed to consist of guanine crystals (Land 1985; Barth 2001). When we wanted to measure the thickness of the crystals we found that in standard-stained ultrathin sections for EM (using uranyl acetate and lead citrate), the crystals seemed to be dissolved, leaving only empty cavities (Fig. 3b: Ca). These cavities varied in width, making it impossible to estimate the thickness of the crystals they originally contained. The problem of reflective crystal wash-out from iridophores and guanophores was also described by other authors (Kawaguti and Kamishima 1966; Setoguti 1967; Rohrlich and Porter 1972; Seitz 1972; Frese 1978). However, in unstained sections the guanine crystals remained intact (Kawaguti and Kamishima 1966; Setoguti 1967). By leaving ultra-thin EM sections unstained, we also found the crystals intact (Fig. 3c: C), although to variable degrees. But with $57.5 \pm 11.5 \mathrm{~nm}$ 


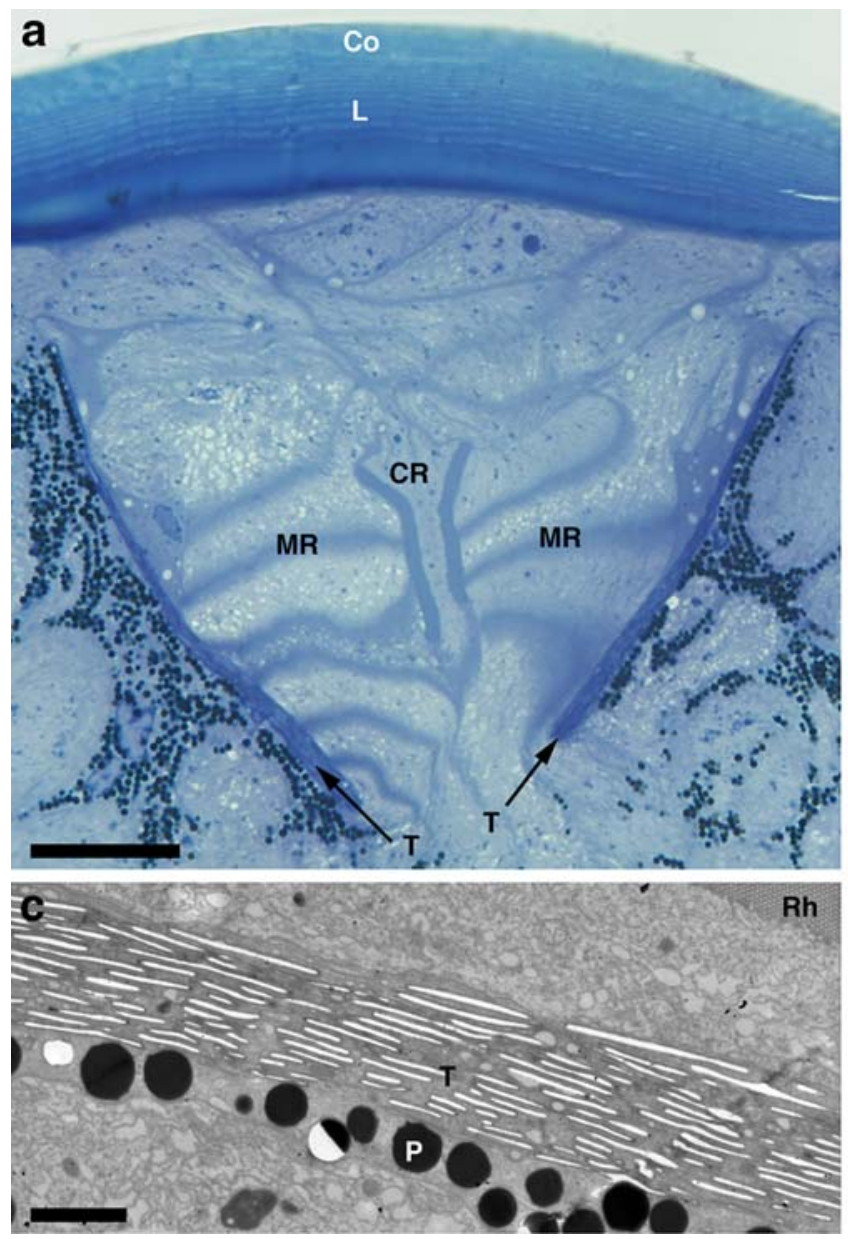

Fig. 2 Cross sections through the postero-median eyes of the spiders Drassodes cupreus $(\mathbf{a}, \mathbf{c})$ and Agelena labyrinthica $(\mathbf{b}, \mathbf{d}) . \mathbf{a}, \mathbf{b}$ Light micrographs of whole eyes. Co cornea, $C R$ central receptors, $L$ lens, $M R$ main receptors, $T$ Tapetum. The microvilli of the main receptors are orthogonal to the plane of the page, those of the central receptors

(mean $\pm \mathrm{SD}$ ) crystal thickness was quite constant. With $114.6 \pm 37.2 \mathrm{~nm}$ the cytoplasmic spaces were somewhat more variable. The measurements were made on 440 crystals and spaces on 22 sections (20 measurements for every section) obtained from four $D$. cupreus spiders.

The arrangement of the layers forming the tapetum in the PM eyes of $A$. labyrinthica was less regular than in D. cupreus in two aspects. (1) The stacks of layers changed their orientation from the central part of the tapetum toward the lateral rim because of the curvature of the tapetum. (2) The layers were sometimes not stacked perfectly parallel on top of each other (Fig. 2b, d).

\section{Reflective material}

As observed on semi-thin sections between crossed polarizers, the obviously birefringent crystals forming the tapetum in the PM eyes of D. cupreus were still in place
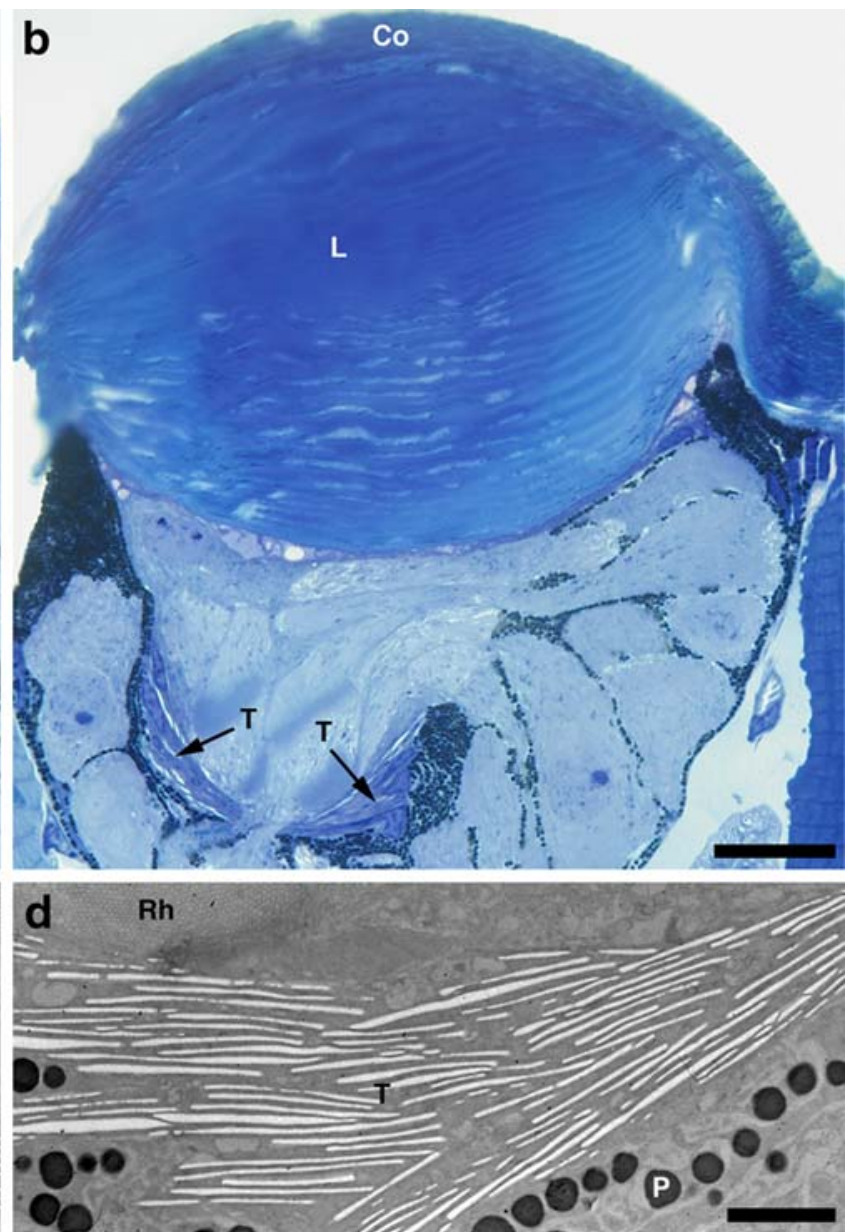

are parallel to it. Sections stained with methylene blue. Calibration bar $20 \mu \mathrm{m}$. c, d Electron micrographs of tapetum. Sections stained with uranyl acetate and lead citrate. $R h$ rhabdom, $P$ pigment granules, $T$ tapetum. Calibration bar $2 \mu \mathrm{m}$

after the process of fixation and histological sectioning. This could be seen best in unstained sections, but also in sections stained with methylene blue the crystals were still visible (Fig. 4a). The same was observed in the PM eyes of A. labyrinthica (data not shown). Thus, such sections were suitable for histochemical approaches to identify the nature of the reflective tapetal material. In animal multilayer reflectors, the high-refractive index layers can be composed of guanine, hypoxanthine, chitin or protein (Land 1972, 1981; Herring 1994). In spiders they have been claimed to consist of guanine crystals (Land 1985; Barth 2001; Schwab et al. 2002). To obtain reliable refractive index values for computer modeling, we tested this view by treating semi-thin or thick cross sections through the PM eyes of D. cupreus with a histochemical procedure previously used to identify guanine. Schmorl's cyanoferrat method (Waldmann 1962) slowly stained the tapetal material with a light blue color (Fig. 4b). According to the 


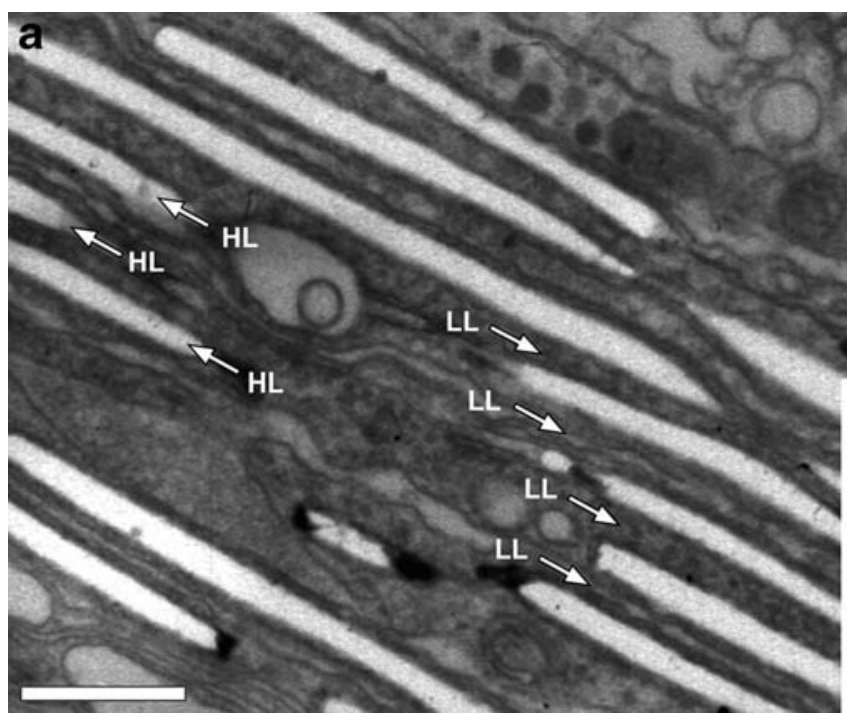

Fig. 3 Electron micrographs of cross-sections through the tapetum of the postero-median eyes of D. cupreus. a, b Sections stained with uranyl acetate and lead citrate. $C a$ cavities, $C P$ cytoplasm, $H L$ high refractive index layer, $L L$ low refractive index layer, $M V$ microvilli of the main receptors. Calibration bar $0.5 \mu \mathrm{m}$ (a) and $0.1 \mu \mathrm{m}(\mathbf{b})$. c
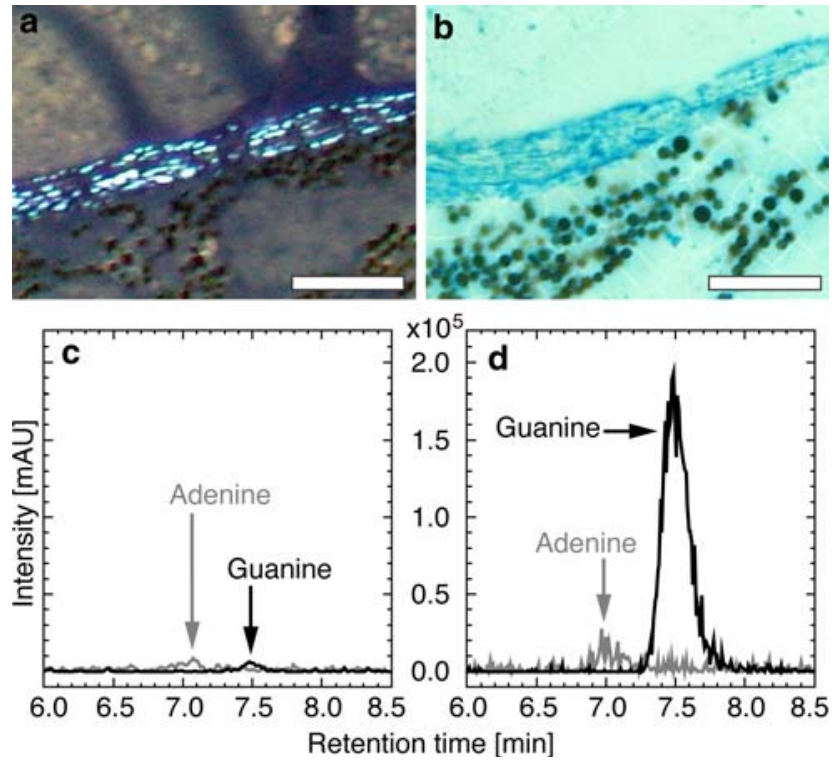

Fig. 4 Properties of the crystals in the high refractive index layer of the tapetum in the postero-median eyes of D. cupreus. a Light micrograph of a cross-section viewed between incompletely $\left(<90^{\circ}\right)$ crossed polarizers. Under these conditions, the material of the high refractive index layer appears bright due to birefringence while the surrounding tissue is dark but still visible. Calibration bar $10 \mu \mathrm{m}$. b Light micrograph of a cross-section stained with Schmorl's cyanoferrat method. With (unpolarized) brightfield illumination the crystals appear blue. Calibration bar $10 \mu \mathrm{m}$. c, d Mass spectrometric data of the purines adenine and guanine in extracts of different eye types of D. cupreus. $Y$-axis indicates absorption (arbitrary units). c Extract of two principal eyes (antero-median eyes lacking a tapetum). d Extract of six secondary eyes (two postero-median, two postero-lateral, two antero-lateral eyes containing a tapetum). Note the large difference between guanine-to-adenine ratios in the two eye types
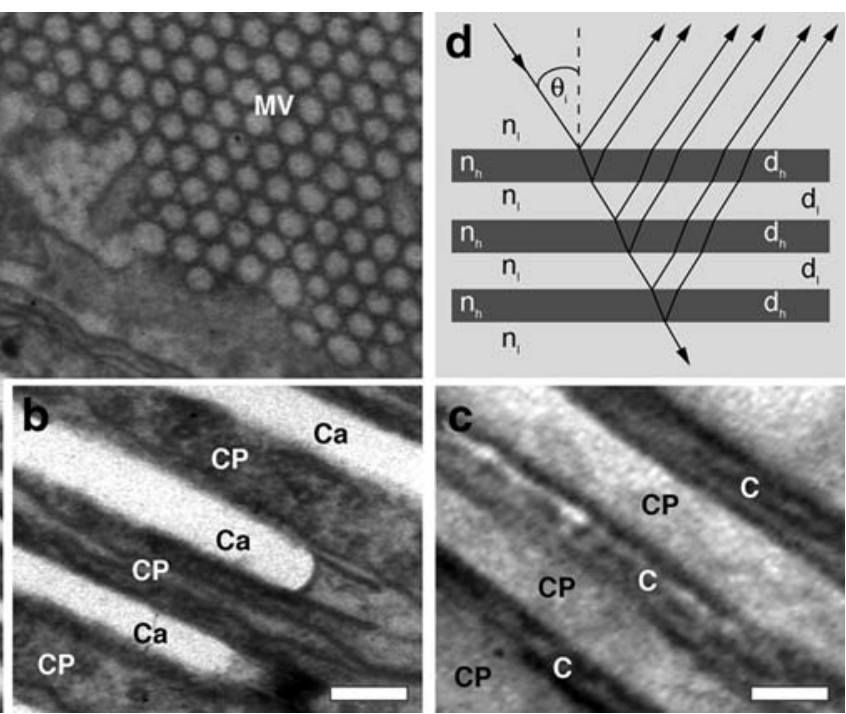

Unstained section. $C$ crystals, $C P$ cytoplasm. Calibration bar $0.1 \mu \mathrm{m}$. d Schematic drawing of a multilayer reflector. Arrowed lines indicate incident, reflected and transmitted light beams, $n_{\mathrm{h}}, n_{1}$ refractive indices of high and low refractive index layers, $d_{\mathrm{h}}, d_{1}$ thicknesses of high and low refractive index layers, $\theta_{\mathrm{i}}$ angle of light incidence

protocol, guanine should indeed be stained light blue very slowly by this method, whereas uric acid and urates are immediately stained dark blue, xanthine is gradually stained blue, and other purines like adenine and hypoxanthine show no effect at all (Waldmann 1962).

To supplement the histochemical test, we also used a HPLC/MS approach to assess the nature of reflective tapetal material. The rationale behind this analysis is that in ordinary tissues the relative amount of guanine and adenine (G/A ratio) is constant for different organs of an organism (Chargaff 1963) because each cell shares the same DNA. Tissues containing additional, non-DNA guanine are expected to show increased G/A ratios. All five samples of tissue without tapetal material had G/A ratios $<1$ (ranging from 0.5 to 0.9$)$. All except one of seven samples containing eyes with tapetum showed G/A ratios $>1$ (ranging from 2.0 to 16.3; exception: 0.4 ), which is significantly higher than in non-tapetal tissue (Mann-Whitney $U$ test, $n=12, z=-2.034, P=0.042)$. These data are exemplified for the extracts of two principal eyes (Fig. 4c) and of six secondary eyes (Fig. 4d).

Thus, as indicated by both tests, the high-refractive index layers of spider tapeta seem indeed to consist of guanine crystals.

\section{Optical properties}

Light reflected by the PM eyes of $D$. cupreus is partially polarized, containing a strong linear component. Any circular components are invisible to both the polarimeter 

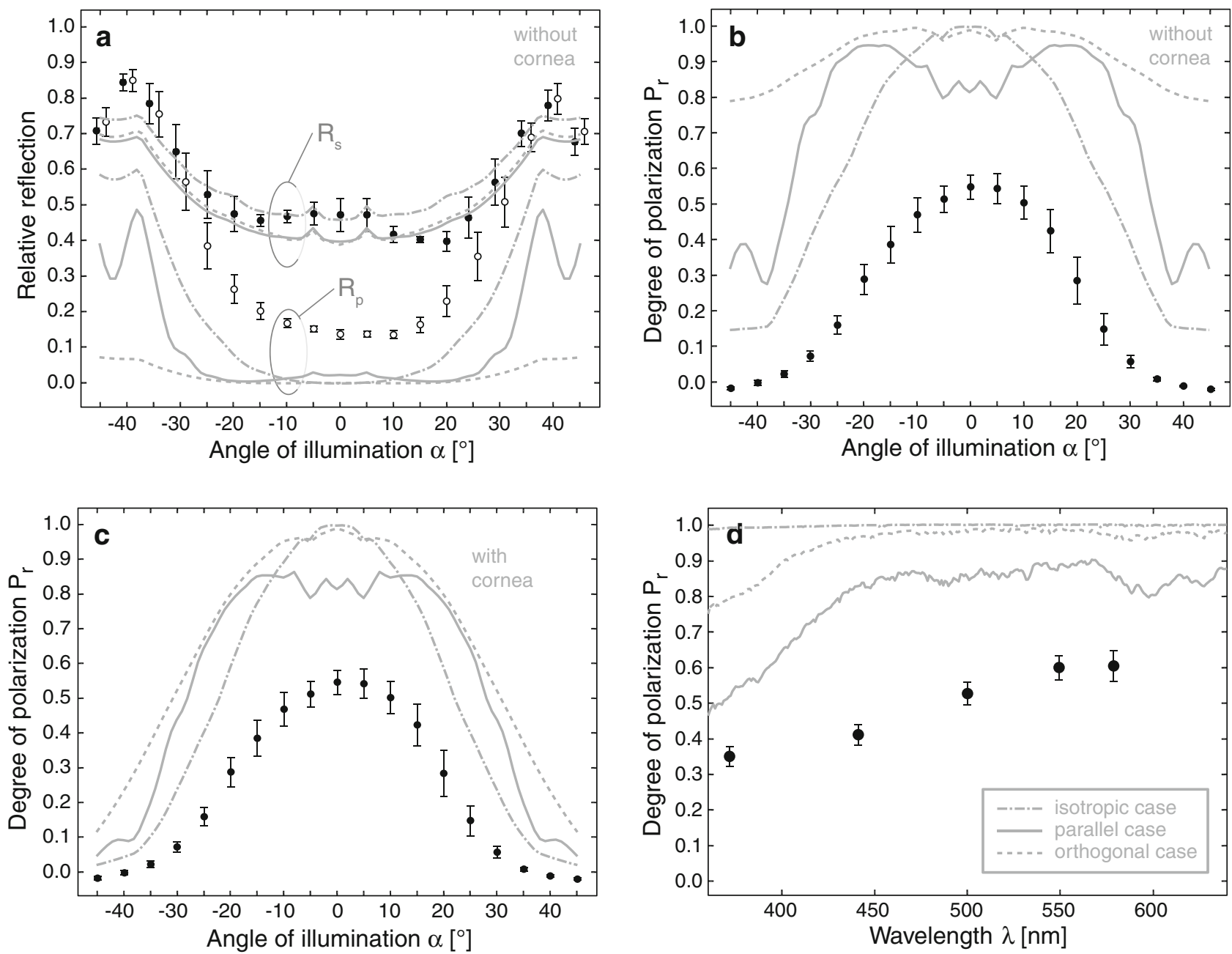

Fig. 5 Intensity and polarization of light reflected by the tapetum of the postero-median eyes of $D$. cupreus at different angles (a-c $\left.\lambda_{\text {med }}=550 \mathrm{~nm}\right)$ and wavelengths $\left(\mathbf{d} \alpha=0^{\circ}\right)$ of incident light. Symbols and lines indicate measured and modeled data, respectively. Dashed-dotted lines refer to isotropic model, continuous lines and dashed lines to birefringent model with purine molecules oriented parallel and orthogonal to the broad side of the crystal platelets, respectively. a Intensity of reflection $R_{\mathrm{s}}$ (maximal reflection, filled circles) and $R_{\mathrm{p}}$ (minimal reflection, empty circles) obtained from whole-eye measurements (see Fig. 1d). The upper family of curves

gives modeled data for $R_{\mathrm{S}}$, the lower one for $R_{\mathrm{p}}$. Modeled data do not take in account light reflection at the air/cornea interface, but curves including cornea reflection are almost identical (not shown). b Degree of polarization $P_{\mathrm{r}}$ calculated from measured (filled circles) and modeled (lines) $R_{\mathrm{s}}$ and $R_{\mathrm{p}}$ values in a. Modeled data do not include effects of cornea reflection. c Degree of polarization $P_{\mathrm{r}}$ at different angles of incidence. Modeled data include effects of cornea reflection. d Degree of polarization $P_{\mathrm{r}}$ at different wavelengths. Modeled data including and excluding cornea reflection are identical

system and the microvillar photoreceptors of the PM eyes. When observing the reflection for different planes of polarization by using a linear polarizer (analyzer) in the exit beam path of the microscope, it is strongest for e-vector orientation parallel to the long axis of the eye $\left(R_{\mathrm{S}}\right)$ and weakest for e-vector orientation orthogonal to it $\left(R_{\mathrm{p}}\right)$ (Fig. 1b). If the eye is illuminated with white light, the former reflection appears bright blue to white, whereas the latter is of dark blue color. The intensity of reflected light was measured with a radiometer for different e-vector orientations, and from the reflection intensities $R_{\mathrm{s}}$ and $R_{\mathrm{p}}$, the degree of polarization $P_{\mathrm{r}}$ was calculated (see "Materials and methods").

Reflection intensities were measured under two aspects. (1) With an angle of illumination $\alpha$ varying between $-45^{\circ}$ and $+45^{\circ}$ and a constant wavelength $\left(\lambda_{\text {med }}=550 \mathrm{~nm}\right)$. (2) With varying wavelength $\lambda$ under a constant angle of illumination $\alpha=0^{\circ}$. Note that the angles $\alpha$ given here are relative to the symmetry plane along the long axis of the eye and do not indicate angles of incidence relative to the multilayer stacks. Thus, assuming the two multilayer stacks to be ideally flat with an angle of $95^{\circ}$ between the sides, an angle of $+15^{\circ}$ would correspond to an incidence angle of either $27.5^{\circ}\left(\theta_{\mathrm{i}, 1}=42.5^{\circ}-\alpha\right)$ or $57.5^{\circ}\left(\theta_{\mathrm{i}, 1}=42.5^{\circ}-\alpha\right)$ on the first stack (depending on whether the left or right side is defined as the "first" stack) and $67.5^{\circ}$ or $37.5^{\circ}$ on 

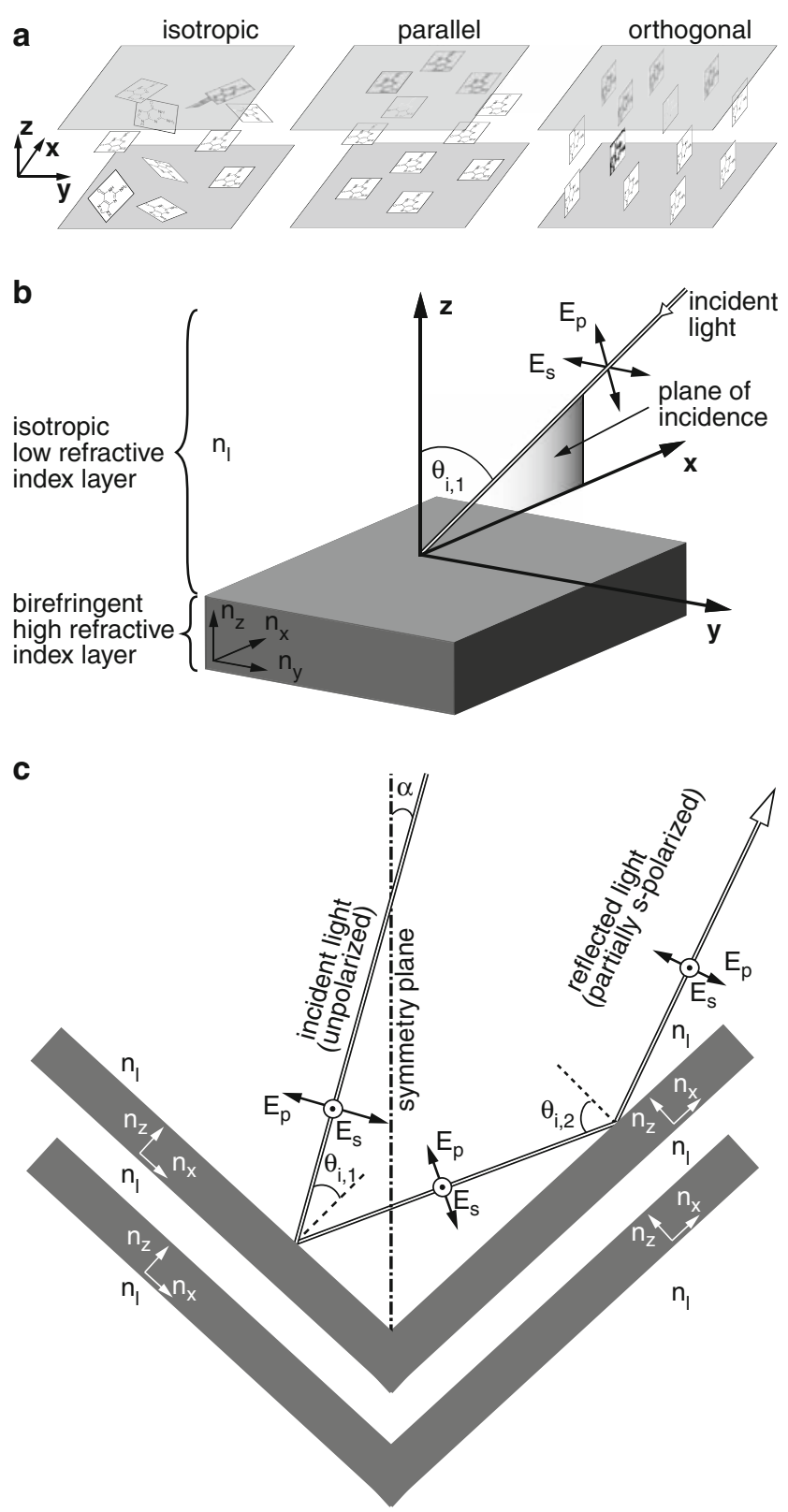

the second stack $\left(\theta_{\mathrm{i}, 2}=95^{\circ}-\theta_{\mathrm{i}, 1}\right.$; see Figs. 1c, 6c $)$. Measurements were performed on $8 \mathrm{PM}$ eyes of 4 spiders for the angle-dependent measurements, and on 9 PM eyes of 5 spiders for the wavelength-dependent measurements.

\section{Angle-dependent measurements}

Figure 5a (circle symbols) shows the reflection of green light $\left(\lambda_{\text {med }}=550 \mathrm{~nm}\right)$ from PM eyes of $D$. cupreus for both orientations of polarization $\left(R_{\mathrm{s}}, R_{\mathrm{p}}\right)$ as functions of the angle of illumination $\alpha$. The strongest reflection for both e-vector orientations parallel $\left(R_{\mathrm{s}}\right)$ and orthogonal $\left(R_{\mathrm{p}}\right)$ to the long axis of the eye was obtained with illumination angles $\alpha$ of $-40^{\circ}$ and $+40^{\circ}\left(\bar{R}_{s}=0.83 \pm 0.07, \bar{R}_{p}=\right.$
Fig. 6 Parameter definitions for modeling reflections and polarization properties of the tapetum in the postero-median eyes of D. cupreus. a Three possible orientations of guanine molecules in the crystal platelets. Planar molecules (molecule plane in white) can be oriented randomly, parallel or orthogonal to the broad face of the crystal platelets (gray). b A beam of light entering from an isotropic low refractive index layer $\left(n_{1}\right)$ to a birefringent high index layer $\left(n_{\mathrm{x}}, n_{\mathrm{y}}, n_{\mathrm{z}}\right)$. The orthogonal optic axes of the birefringent high index layer coincide with the layer axes $(x, y, z)$. If the incidence plane is the $x-z$-plane, light polarized along the $y$-axis has its electric field orthogonal to the plane of incidence $\left(E_{\mathrm{s}}\right)$ and is called s-polarized light. Light polarized in the $x-z$-plane has its electric field parallel to the plane of incidence $\left(E_{\mathrm{p}}\right)$ and is called p-polarized light. Adapted from Weber et al. (2000). c Schematic drawing of the modeled tapetum used to calculate reflection and polarization. The drawing plane corresponds to the plane of incidence $(x-z$-plane), the $y$-axis is oriented orthogonal to the drawing plane, $n_{\mathrm{y}}$ refractive indices are, therefore, not shown. High index layers are shown in gray. The decreasing length of the double arrow $E_{\mathrm{p}}$ representing the direction of the electric field parallel to the plane of incidence indicates qualitatively the increasing s-polarization

$0.84 \pm 0.07)$ (mean $\pm \mathrm{SD})$. In this case, reflection was essentially from one side of the tapetum only, with normal light incidence on either reflector. Minimal reflection for $R_{\mathrm{s}}$ was at about $-15^{\circ}\left(\bar{R}_{s}=0.47 \pm 0.04\right)$ and $+20^{\circ}\left(\bar{R}_{s}=\right.$ $0.41 \pm 0.06)$, and for $R_{\mathrm{p}}$ between $0^{\circ}$ and $+10^{\circ}\left(\bar{R}_{p}=\right.$ $0.14 \pm 0.02)$. Differences between left and right eyes are not significant (repeated measures ANOVA, $R_{\mathrm{s}}: F_{1,3}=$ $\left.2.042, P=0.248 ; R_{\mathrm{p}}: F_{1,3}=0.314, P=0.615\right)$, and, therefore, reflection intensities from left and right eyes of each individual were averaged.

The highest degrees of polarization $P_{\mathrm{r}}$ were observed with zenithal illumination $\left(\alpha=0^{\circ}\right.$; Fig. 5c). $P_{\mathrm{r}}$ values of up to $0.67\left(\bar{P}_{r}=0.55 \pm 0.07\right)$ were obtained if the whole eye was illuminated (see Fig. 1d). For partial illumination (Fig. 1e), we measured $P_{\mathrm{r}}$ values of up to 0.89 . Previous authors give a $P_{\mathrm{r}}$ value of 0.68 for the PM eyes of D. cupreus (Dacke et al. 2001). With increasing illumination angles, the $P_{\mathrm{r}}$ values decreased reaching a minimum at $\pm 45^{\circ}$. At these angles, the $P_{\mathrm{r}}$ values even became slightly negative because $R_{\mathrm{p}}$ exceeded $R_{\mathrm{s}}$.

\section{Wavelength-dependent measurements}

The polarizing properties of the tapetum at different wavelengths $\left(\lambda_{\text {med }}\right)$ were examined under zenithal illumination $\left(\alpha=0^{\circ}\right)$. The degree of polarization $P_{\mathrm{r}}$ was weakest at $370 \mathrm{~nm}\left(\bar{P}_{r}=0.35 \pm 0.06\right)$ increasing toward longer wavelengths and reaching $\bar{P}_{r}=0.60 \pm 0.10$ at $580 \mathrm{~nm}$ (Fig. 5d).

\section{Mathematical modeling}

For modeling the reflective and polarizing properties of the PM eyes of D. cupreus, we assumed the two multilayer stacks forming the sides of the tapetum to be exactly flat 
with an angle of $95^{\circ}$ between them. In the model, each of the two multilayer stacks contains 20 high refractive index layers embedded in cytoplasm. Crystal thickness values were computed randomly, following a normal distribution based on the mean value and standard deviation measured in unstained EM sections. Cytoplasmic space thicknesses were also computed randomly, following a log-normal distribution since the distribution of measured space thickness was skewed. We modeled 200 different stacks, and the resulting reflection and polarization data were averaged.

The refractive index for the cytoplasmic spaces was set to that of water, $n_{1}=1.33$ (Land 1972). For the birefringent crystals (see Fig. 4a), we had to use three different refractive index values associated with the three crystal axes. Thus, the tapetum actually consists of birefringent multilayer reflectors. The refractive indices applied (1.85, 1.80 and 1.46 for all wavelengths) are actually not the values for pure guanine but of a mixture of $76 \%$ guanine and $24 \%$ hypoxanthine, as found in crystal platelets of the scales of the herring (Greenstein 1973). Crystal platelets from other fish differ in this ratio but guanine is always the main component (Greenstein 1973) and the refractive indices always fall between the limits of pure guanine (1.93, 1.91 and 1.468) and pure hypoxanthine $(1.85,1.78$ and 1.42). Applying different triplets of refractive indices in this range for comparison, did not significantly change the reflection and polarizing properties of the model tapetum (data not shown).

However, it is essential how the planar purine molecules, and, therefore, the crystal axes, are oriented with respect to the surface of the crystal platelets. In fish scales, the planar purine molecules lie essentially parallel to the broad face of the crystals in the $x-y$-plane (henceforth called the "parallel case"; Fig. 6a, middle sketch), resulting in $n_{\mathrm{x}}=1.85, n_{\mathrm{y}}=1.80$ and $n_{\mathrm{z}}=1.46$ (Greenstein 1973; Levy-Lior et al. 2008). For normal light incidence on the plate $\left(\theta_{\mathrm{i}}=0^{\circ}\right)$ along the $z$-axis, reflection is high for light oscillating both along the $x$-axis and the $y$-axis since the refractive index differences between crystal and cytoplasm is high for both e-vector orientations (1.85 vs. 1.33 along the $x$-axis, 1.80 vs. 1.33 along the $y$-axis). This makes the crystal a good reflector. In contrast to fish scales, synthetic crystalline platelets of organic substances with planar molecules mostly consist of molecules oriented orthogonal to the broad face of the platelets in the $\mathrm{y}-\mathrm{z}$ plane ("orthogonal case"; Fig. 6a, right sketch), a condition that is also obtained when guanine-hypoxanthine mixtures are crystallized in vitro (Greenstein 1973; LevyLior et al. 2008). In the orthogonal case $\left(n_{\mathrm{x}}=1.46\right.$, $\left.n_{\mathrm{y}}=1.80, n_{\mathrm{z}}=1.85\right)$, reflection for normal incidence along the $z$-axis remains high for light oscillating along the $y$-axis, but for polarization along the $x$-axis, reflection is reduced because the refractive index difference along the $x$ axis is small ( $n_{\mathrm{x}}=1.46$ vs. $\left.n_{1}=1.33\right)$. This results in polarization of reflected light incident normal to the surface of the reflector.

For obliquely incident light with the plane of incidence in the $x-z$-plane (Fig. 6b), light polarized along the $y$-axis corresponds to s-polarization (e-vector orthogonal to plane of incidence) and the reflection only depends on $n_{\mathrm{y}}$, whereas the reflection of p-polarized light (e-vector parallel to plane of incidence) depends on both $n_{\mathrm{x}}$ and $n_{\mathrm{z}}$ (Weber et al. 2000). For the orthogonal case, s-polarized light is strongly reflected whereas for p-polarized light reflection is weak. For the parallel case, this difference is much smaller.

We ran the model for both cases of molecule orientation. The plane of illumination was always the $x$-z-plane; the long axis of the model eye corresponds to the $y$-axis (Fig. 6c). We also ran the model for isotropic high index layers with $n_{\mathrm{x}}=n_{\mathrm{y}}=n_{\mathrm{z}}=1.83$ (Fig. 6a, left sketch), a model previously chosen by several authors (e.g., Denton and Land 1971; Land 1972; McKenzie et al. 1995).

The model simulated reflection and polarization data under the same conditions as measured optically: (1) with angles of illumination $\alpha$ varying between $-45^{\circ}$ and $+45^{\circ}$ and a constant wavelength $\lambda$ (averaged between 525 and $575 \mathrm{~nm}$ ). (2) With varying wavelength $\lambda$ at a constant angle of illumination $\alpha=0^{\circ}$. Because of the aforementioned ambiguity concerning incidence angles $\theta_{\mathrm{i}, 1}$ and $\theta_{\mathrm{i}, 2}$ with respect to the angle of illumination $\alpha$ (refer to Fig. 1c), we calculated both possibilities and averaged the resulting reflection intensities.

The angle-dependent polarization data of the model (Fig. 5b) show a roughly bell-shaped curve in the isotropic case. In the birefringent cases, the shape of the curve is more complicated, with multiple maxima and minima. In the orthogonal case, a substantial polarization is maintained over the whole range of incidence angles because $R_{\mathrm{s}}$ remains low for any angle of incidence. In the parallel case, $P_{\mathrm{r}}$ values rapidly drop for incidence angles larger than $\pm 20^{\circ}$.

Polarization by reflection at the interface between air $\left(n_{\mathrm{a}}=1.00\right)$ and cornea $\left(n_{\mathrm{c}}=1.40\right)$ (Blest et al. 1981) attenuates the polarizing effect of the tapetum at all illumination angles $\alpha$ (except $0^{\circ}$ ) since s-polarized light is reflected away and, therefore, light entering the eye is already p-polarized to some degree, depending on $\alpha$. Figure 5c shows the polarization $P_{\mathrm{r}}$ of reflected light for different $\alpha$ values including this cornea effect. The cornea was assumed to be flat; including the slight curvature had no substantial influence (data not shown). The cornea effect is strongest for large $\alpha$ values approaching Brewster's angle between air and cornea of $54^{\circ}$. The cornea effect results in rather similar, roughly bell-shaped $P_{\mathrm{r}}$ functions for all, the isotropic, parallel and orthogonal cases of the model. 
The spectral data of the model (Fig. 5d) show that the otherwise quite constant degree of polarization $P_{\mathrm{r}}$ decreases below $450 \mathrm{~nm}$ for the birefringent cases of the model. In contrast, in the isotropic case, polarization remains at extremely high levels (almost 1) over the whole range of wavelengths evaluated. Comparing modeled angle-dependent $P_{\mathrm{r}}$ values of reflected UV (325-375 nm) and green light $(475-525 \mathrm{~nm})$, we found that $P_{\mathrm{r}}$ is always higher in the green for all cases of the model and all angles of illumination (data not shown).

\section{Discussion}

Angular properties of the tapetum

For the reflections $R_{\mathrm{s}}$, measured and modeled data agree fairly well. Both show maxima at large angles of illumination $\alpha$ and broad minima for small angles (Fig. 5a; closed symbols and upper family of curves). Regarding the $R_{\mathrm{p}}$ values, the overall U-shaped course of the measured data is compatible with the model for the isotropic and parallel case (Fig. 5a; open symbols, and continuous and dashdotted curves), but measured reflections are always stronger than modeled. This could be an effect of the unavoidable, unpolarized reflection of incident light on the cornea surface. However, the strong increase of measured $R_{\mathrm{p}}$ values with increasing angles of illumination disagrees with the orthogonal case, where reflection of p-polarized light should remain low for all angles (Fig. 5a; dashed curve).

Regarding the degrees of polarization $P_{\mathrm{r}}$, both modeled and measured data follow bell-shaped angular functions. But the model generally predicts substantially higher values than actually measured optically. This is not unexpected for the following reasons. (1) Measured $P_{\mathrm{r}}$ values are lower than the real ones because the unpolarized corneal reflection influenced the reflection data, thus reducing the $P_{\mathrm{r}}$ values calculated from them up to an estimated $20 \%$ depending on the preparation and the angle of illumination. (2) The modeled $P_{\mathrm{r}}$ values are higher than the real ones since the model is based on two perfectly flat multilayer stacks oriented at exactly $95^{\circ}$, an ideal condition which is probably not met in reality. In addition, the model excludes estimations of selective absorption of s-polarization by the main receptors.

In addition to the modeled data presented above, we have varied some parameters, i.e., we used different refractive index triplets by varying the guanine/hypoxanthine ratio, we tried every possible permutation of $n_{\mathrm{x}}, n_{\mathrm{y}}$ and $n_{\mathrm{z}}$ and changed the angle between the two sides of the tapetum. None of these changes led to a closer fit between model and measurements (data not shown).
The presence of two flat multilayer reflectors oriented roughly orthogonal to each other suggested to us that the polarizing action of the tapetum is due to polarization by double reflection. Using a computer model of the tapetum, the polarization observed in the intact eye could indeed be simulated. Differences between observed and modeled degrees of polarization can be explained by polarizationreducing influences in the optical measurements, and the assumption of ideal geometrical conditions in the model. We, therefore, believe that apart from polarization by reflection no additional polarizing mechanisms need to be invoked.

\section{Spectral properties of the tapetum}

With a decrease of polarization $P_{\mathrm{r}}$ toward short wavelengths, both measured and modeled data for the birefringent cases qualitatively agree and are also in accordance with the observation that the tapetal reflection viewed through a polarizer with the e-vector transmission axis orthogonal to the eye long axis appears dark blue (Fig. 1b). As in the angular case, the model predicts higher $P_{\mathrm{r}}$ values than actually measured, which can be explained by the same polarization-reducing effects.

\section{Orientation of guanine molecules}

For the model tapetum, we have considered three possibilities of orientation for the guanine molecules composing the tapetum, i.e., parallel, orthogonal or random with respect to the broad face of the crystal platelets. Do our data indicate which one of these options is actually implemented in the PM eyes of $D$. cupreus? The $P_{\mathrm{r}}$ values of reflected light measured for different illumination angles $\alpha$ follow a bell-shaped function. All modeled $P_{\mathrm{r}}$ functions are also largely bell-shaped, provided that the reflection of light at the air/cornea interface is included (Fig. 5c), not allowing any conclusion. However, the birefringence of guanine crystals, which can be directly observed in histological sections between crossed polarizers (Fig. 4a) rules out the presence of isotropic high index layers. The following data support the parallel rather than the orthogonal case of the model. (1) The strong increase of measured $R_{\mathrm{p}}$ values with increasing angles of incidence contradicts the orthogonal case (Fig. 5a). (2) As found in scales of fish, in other biological reflectors the planar purine molecules in reflective crystals are also oriented parallel to the broad face of the crystal platelets (Greenstein 1973; Levy-Lior et al. 2008).

Although in the orthogonal case reflected light is polarized slightly more strongly (Fig. 5c; model data in $\pm 15^{\circ}$ range), parallel molecular orientation makes the tapetum a better reflector for normal incident light (Fig. 5a; 
model data at $\pm 45^{\circ}$ ). Spiders were among the first predators on land and can be traced back to the middle Devonian period according to the fossil record (Coddington and Levi 1991). This was before the evolution of flying insects (Brodsky 1994), which many modern spiders entrap in their webs. Hence, prehistoric spiders probably hunted their prey much like hunting lycosid spiders of today and developed their tapeta to allow them to take advantage of nocturnal conditions (Schwab et al. 2002). Thus, the tapeta in spider eyes probably evolved to reflect as much light as possible and not to polarize it as strongly as possible.

\section{Ecophysiological considerations}

The PM eyes of $D$. cupreus are non-imaging visual systems because the lenses are reduced to flat windows directly overlying the photoreceptors and because screening pigment optically separating the photoreceptors from each other is missing. All photoreceptors must, therefore, have extremely wide, overlapping visual fields. Using an optical method, the visual fields of the PM eyes were found to be zenith-centered and $120^{\circ}$ to $130^{\circ}$ wide (Dacke et al. 1999, 2001). Spatial integration over a large part of the sky can make polarized skylight navigation largely insensitive to disturbances of the celestial polarization pattern by clouds or terrestrial objects (Labhart 1999). But averaging different e-vector orientations within the field of view and including unpolarized light from clouds can result in significant reduction of the effective degree of polarization. Reading such weak polarization signals in the sky requires high polarization sensitivity. The polarizing tapetum of $D$. cupreus boosts the inherent, microvilli-based polarization sensitivity of most photoreceptors by attenuating light of the e-vector orientation to which the receptors are least sensitive. Since the two PM eyes are oriented approximately orthogonal to each other (Dacke et al. 1999) their main receptors are tuned to mutually orthogonal e-vector orientations. Antagonistic interaction between the two PM eyes may, therefore, further enhance the polarization sensitivity of the system, in a similar way as in the DRA of insects (Labhart and Meyer 1999, 2002; Wehner and Labhart 2006).

Both measured and modeled data indicate that the polarizing effect of the tapetum is stronger for green than for UV light. In addition, both light intensity and polarization in the sky are higher at $500 \mathrm{~nm}$ than at $350 \mathrm{~nm}$ (Coulson 1988), even under twilight conditions when the spiders are most active (Dacke et al. 1999; Cronin et al. 2006; Johnson et al. 2006). One would thus expect that $D$. cupreus uses the green-receptors for polarized skylight navigation.

Light absorbed by the photoreceptors is a mixture of unreflected, singly and doubly reflected light. Therefore, the degree of polarization effectively seen by the photoreceptors will be somewhat lower than the degree of polarization we measured and calculated in our model, since measurement and model both take into account doubly reflected light only. The same is true regarding the wavelength spectrum, which will be slightly shifted toward the spectrum of incident light.

\section{Comparison with the PM eyes of A. labyrinthica}

As for D. cupreus, e-vector navigation was also demonstrated by behavioral tests for the funnel-web spider $A$. labyrinthica (Görner 1958, 1962). However, as shown by eye occlusion experiments, polarization vision in this spider is mediated by the principal eyes and the PM eyes are not involved (Görner 1958). This agrees with the structure of the PM eyes, which do not support polarization sensitivity. (1) The rhabdoms of the photoreceptors exhibit a disordered arrangement. (2) The two reflectors forming the canoe-shaped tapetum are curved, i.e., the orientation of the multilayer stacks is not constant. This arrangement and the presence of a proper lens suggests that light entering the eye will be refracted such that for every beam of light the incidence angle $\theta_{\mathrm{i}}$ on the curved tapetum will be close to $0^{\circ}$, meaning that it will be reflected back in an unpolarized state. This view is in accordance with optical measurements of reflected light in the PM eyes of A. labyrinthica showing a degree of polarization of just 0.09 (Dacke et al. 2001). In line with the eye occlusion experiments in A. labyrinthica (Görner 1958, 1962), its close relative A. gracilens has a ventral polarization-sensitive retinal area (see "Introduction") in the principal eyes (Schröer 1974).

Acknowledgments We thank Dr. Laurent Bigler for running the HPLC/MS analysis of the spider eyes and Dr. Doekele Stavenga for critically reading an earlier version of the manuscript.

\section{Appendix}

The reflectance $\Gamma$ of a birefringent multilayer stack with $\mathrm{M}$ layers and $\mathrm{M}+1$ interfaces was calculated for $\mathrm{p}$ - and $\mathrm{s}$ polarized light recursively as follows. The derivation of these formulae can be found in Orfanidis (2008).

$\Gamma_{i}=\frac{\rho_{i}+\Gamma_{i+1} e^{-2 j \delta_{i}}}{1+\rho_{i} \Gamma_{i+1} e^{-2 j \delta_{i}}}, \quad i=M, M-1, \ldots, 1$.

This recursion was initialized with $\Gamma_{M+1}=\rho_{M+1}$.

In Eq. $3, j$ the imaginary unit, $\rho_{\mathrm{i}}$ the transverse reflection coefficient and $\delta_{\mathrm{i}}$ the complex phase thickness of the $i$ th layer. The complex phase thicknesses $\delta_{\mathrm{i}}$, depending on the state of polarization, can be calculated as: 


$$
\begin{gathered}
\delta_{\mathrm{i}}=\left\{\begin{array}{l}
\frac{2 \pi}{\lambda} l_{\mathrm{i}} n_{\mathrm{i} 1} \sqrt{1-\frac{n_{\mathrm{a}}^{2} \sin ^{2}\left(\theta_{\mathrm{a}}\right)}{n_{i 3}^{2}}} \text { (p-polarization) } \\
\frac{2 \pi}{\lambda} l_{\mathrm{i}} n_{\mathrm{i} 2} \sqrt{1-\frac{n_{\mathrm{a}}^{2} \sin ^{2}\left(\theta_{\mathrm{a}}\right)}{n_{\mathrm{i} 2}^{2}}} \text { (s-polarization) }
\end{array}\right. \\
\mathrm{i}=1,2, \ldots, M+1 .
\end{gathered}
$$

where $n_{\mathrm{i} 1}, n_{\mathrm{i} 2}$ and $n_{\mathrm{i} 3}$ are the refractive indices and $l_{\mathrm{i}}$ the thickness of the $i$ th layer. $\theta_{\mathrm{a}}$ is the angle of incidence on the first interface and $n_{\mathrm{a}}$ the refractive index of the incident medium.

The transverse reflection coefficients $\rho_{\mathrm{i}}$ of Eq. 3 can be calculated as:

$\rho_{\mathrm{i}}=\frac{\eta_{i-1}-\eta_{i}}{\eta_{i-1}+\eta_{i}}, \quad i=a, 1,2, \ldots, M, b$.

$\eta_{\mathrm{i}}$ are the transverse refractive indices, which depend on the state of polarization:

$\eta_{\mathrm{i}}= \begin{cases}\frac{n_{\mathrm{i}} n_{\mathrm{i} 3}}{\sqrt{n_{\mathrm{i} 3}^{2}-n_{\mathrm{a}}^{2} \sin ^{2}\left(\theta_{\mathrm{a}}\right)}} & (\mathrm{p}-\text { polarization }) \\ \sqrt{n_{\mathrm{i} 2}^{2}-n_{\mathrm{a}}^{2} \sin ^{2}\left(\theta_{\mathrm{a}}\right)} & (\mathrm{s}-\text { polarization })\end{cases}$

where the index $a$ stands for the incident medium and $b$ for the substrate (the medium after the last interface of the stack).

\section{References}

Barth FG (2001) Sinne und Verhalten: aus dem Leben einer Spinne. Springer, Berlin

Blest AD, Hardie RC, McIntyre P, Williams DS (1981) The spectral sensitivities of identified receptors and the function of retinal tiering in the principal eyes of a jumping spider. J Comp Physiol A $145: 227-239$

Brodsky AK (1994) The evolution of insect flight. Oxford University Press, Oxford

Chargaff E (1963) Essays on nucleic acids. Elsevier, Amsterdam

Coddington JA, Levi HW (1991) Systematics and evolution of spiders (Araneae). Annu Rev Ecol Syst 22:565-592

Coulson KL (1988) Polarization and intensity of light in the atmosphere. A. Deepak Publishing, Hampton

Cronin TW, Warrant EJ, Greiner B (2006) Celestial polarization patterns during twilight. Appl Opt 45:5582-5589

Dacke M, Nilsson D-E, Warrant EJ, Blest AD, Land MF, O'Carroll DC (1999) Built-in polarizers form part of a compass organ in spiders. Nature 401:470-473

Dacke M, Doan TA, O'Carroll DC (2001) Polarized light detection in spiders. J Exp Biol 204:2481-2490

Denton EJ, Land MF (1971) Mechanism of reflexion in silvery layers of fish and cephalopods. P R Soc Lond A Mat 178:43-61

Frese J-H (1978) Ultrastructure of the extracutaneous pigment cells in the Plaice (Pleuronectes platessa, L., Teleostei). Cell Tissue Res 195:123-144

Goldsmith TH, Wehner R (1977) Restrictions on rotational and translational diffusion of pigment in the membranes of a rhabdomeric photoreceptor. J Gen Physiol 70:453-490

Görner P (1958) Die optische und kinästhetische Orientierung der Trichterspinne Agelena labyrinthica (Cl.). Z Vergl Physiol $41: 111-153$
Görner P (1962) Die Orientierung der Trichterspinne nach polarisiertem Licht. Z Vergl Physiol 45:307-314

Greenstein LM (1973) Nacreous (Pearlescent) pigments. In: Patton T (ed) Pigment handbook vol 1: properties and economics. Wiley, New York, pp 871-890

Hardie RC (1984) Properties of photoreceptors R7 and R8 in dorsal marginal ommatidia in the compound eyes of Musca and Calliphora. J Comp Physiol A 154:157-165

Henton WW, Crawford FT (1966) The Discrimination of polarized light by the tarantula. Z Vergl Physiol 52:26-32

Herring PJ (1994) Reflective systems in aquatic animals. Comp Biochem Physiol A 109:513-546

Homann H (1971) Die Augen der Araneae. Anatomie, Ontogenie und Bedeutung für die Systematik (Chelicerata, Arachnida). Z Morphol Tiere 69:201-272

Horváth G, Varjú D (2004) Polarized light in animal vision: polarization patterns in nature. Springer, Berlin

Israelachvili JN, Wilson M (1976) Absorption characteristics of oriented photopigments in microvilli. Biol Cybern 21:9-15

Johnson S, Kelber A, Warrant E, Sweeney AM, Widder EA, LLj Lee, Hernández-Andres J (2006) Crepuscular and nocturnal illumination and its effects on color perception by the nocturnal hawkmoth Deilephila elpenor. J Exp Biol 209:789-800

Kawaguti S, Kamishima Y (1966) A supplementary note on the iridophore of the Japanese porgy. Biol J Okayama Univ 12:57-60

Kovoor J, Muñoz-Cuevas A, Ortega-Escobar J (1993) Microanatomy of the anterior median eyes and its possible relation to polarizedlight reception in Lycosa tarentula (Araneae, Lycosidae). B Zool 60:367-375

Labhart T (1999) How polarization-sensitive interneurons of crickets see the polarization pattern of the sky: a field study with an optoelectronic model neurone. J Exp Biol 202:757-770

Labhart T, Meyer EP (1999) Detectors for polarized skylight in insects: a survey of ommatidial specializations in the dorsal rim area of the compound eye. Microsc Res Tech 47:368-379

Labhart T, Meyer EP (2002) Neural mechanisms in insect navigation: polarization compass and odometer. Curr Opin Neurobiol $12: 707-714$

Land MF (1972) The physics and biology of animal reflectors. Prog Biophys Mol Biol 24:75-106

Land MF (1981) Optics and vision in invertebrates. In: Autrum H (ed) Handbook of sensory physiology VII/6B. Springer, Berlin, pp 472-592

Land MF (1985) The morphology and optics of spider eyes. In: Barth FG (ed) Neurobiology of arachnids. Springer, Berlin, pp 53-78

Land MF (2000) Eyes with mirror optics. J Opt A Pure Appl Opt 2:R44-R50

Levy-Lior A, Pokroy B, Levavi-Sivan B, Leiserowitz L, Weiner S, Addadi L (2008) Biogenic guanine crystals from the skin of fish may be designed to enhance light reflectance. Cryst Growth Des 8:507-511

McKenzie DR, Yin Y, McFall WD (1995) Silvery fish skin as an example of a chaotic reflector. Proc R Soc Lond A 451:579-584

Melamed J, Trujillo-Cenóz O (1966) Fine structure of visual system of Lycosa (Araneae-Lycosidae). I. Retina and optic nerve. Z Zellforsch Mik Ana 74:12-31

Nilsson DE, Labhart T, Meyer E (1987) Photoreceptor design and optical properties affecting polarization sensitivity in ants and crickets. J Comp Physiol A 161:645-658

Orfanidis SJ (2008) Electromagnetic waves and antennas. http:// www.ece.rutgers.edu/ orfanidi/ewa/

Ortega-Escobar J, Muñoz-Cuevas A (1999) Anterior median eyes of Lycosa tarentula (Araneae, Lycosidae) detect polarized light: behavioral experiments and electroretinographic analysis. J Arachnol 27:663-671 
Papi F (1955) Astronomische Orientierung bei der Wolfspinne Arctosa perita (Latr). Z Vergl Physiol 37:230-233

Rohrlich ST, Porter KR (1972) Fine structural observations relating to the production of color by the iridophores of a lizard, Anolis carolinensis. J Cell Biol 53:38-52

Schröer WD (1974) Zum Mechanismus der Analyse polarisierten Lichtes bei Agelena gracilens C. L. Koch (Araneae, Agelenidae). I. Die Morphologie der Retina der vorderen Mittelaugen (Hauptaugen). Z Morphol Tiere 79:215-231

Schwab I, Yuen C, Buyukmihci N, Blankenship T, Fitzgerald P (2002) Evolution of the tapetum. Trans Am Ophthal Soc 100:187-200

Seitz KA (1972) Elektronenmikroskopische Untersuchungen an den Guanin-Speicherzellen von Araneus diadematus Clerck (Araneae, Araneidae). Z Morphol Tiere 72:245-262

Setoguti T (1967) Ultrastructure of guanophores. J Ultra Struct R $18: 324-332$

Strutt JWLR (1871) On the light from the sky, its polarization and colour. Philos Mag 41:107-120
Suhai B, Horváth G (2004) How well does the Rayleigh model describe the E-vector distribution of skylight in clear and cloudy conditions? A full-sky polarimetric study. J Opt Soc Am A $21: 1669-1676$

Waldmann H (1962) Zur histochemischen Identifizierung kristalliner Einlagerungen von Harnsäure und Uraten in tierischen und menschlichen Geweben. Mikrochim Acta 50:198-210

Weber MF, Stover CA, Gilbert LR, Nevitt TJ, Ouderkirk AJ (2000) Giant Birefringent optics in multilayer polymer mirrors. Science 287:2451-2456

Wehner R, Labhart T (2006) Polarization vision. In: Warrant EJ, Nilsson D-E (eds) Invertebrate vision. Cambridge University Press, Cambridge, pp 291-348

Wehner R, Bernard GD, Geiger E (1975) Twisted and non-twisted rhabdoms and their significance for polarization detection in the bee. J Comp Physiol A 104:225-245 